\title{
Leishmania Donovani Promastigote: Effect of Adrenergic Agonists and Antagonist on Growth and Lipophosphoglycan Synthesis
}

\author{
Kalipada Kar, ${ }^{1}$ Sujata Kar, ${ }^{1}$ \\ ${ }^{1}$ Department of Physiology, College of Medical Sciences and Teaching Hospital, Bharatpur, Nepal.
}

\begin{abstract}
Background: Leishmania is the causative agent of a spectrum of diseases in human ranging in severity from self-healing cutaneous form to disfiguring mucocutaneous lesion to deadly visceral form of leishmaniasis. The treatment is still suffering from toxicity of the present drug regimen and the emergence of drug resistant leishmaniasis. Successful immunotherapy is yet to develop. It is beneficial to venture the parasite second messenger systems for the development of successful antileishmanial agents. The role of adrenergic receptor agonists and antagonist in the growth of $L$. donovani promastigotes and the synthesis of phosphorylated macromolecules were addressed in this report. Materials and Methods: The present work is entirely experimental. The effect of dibutyryl cAMP (db-cAMP), dibutyryl cGMP (db-cGMP), epinephrine, isoproterenol and propranolol on the growth of $L$. donovani promastigotes using defined medium were studied. Biosynthetically pulse labeling of promastigotes with $\left[{ }^{32} \mathrm{P}\right]$-orthophosphate with or without experimental agents following SDS-PAGE and autoradiography was analyzed. Results: The growth of $L$. donovani promastigotes at $96 \mathrm{~h}$ culture was inhibited by 69\%, 83, 52\% and 95\% with db-cAMP, epinephrine, isoproterenol and propranolol at $100 \mu \mathrm{M}$ each, respectively in compared to control. Multiplication of parasite was stimulated by db-cGMP. The expression of lipophosphoglycan of the parasite was drastically affected in the following order with propranolol>epinephrine $>$ cAMP $>$ isoproterenol at $100 \mu \mathrm{M}$ of each agent for $6 \mathrm{~h}$ exposure to the promastigotes. Conclusion: The growth of the parasite and the synthesis of lipophosphoglycan were significantly more inhibited by epinephrine or propranolol in a dose dependent manner than cAMP or isoproterenol.
\end{abstract}

Keywords: cAMP; cGMP; epinephrine; Leishmania donovani; LPG; propronolol.

\section{INTRODUCTION}

Leishmania donovani is a digenetic hemoflagellate, alternating between a flagellated promastigote form in the hostile midgut of the vector phlebotomine insect and a rudimentary flagellated obligatory intracellular amastigote form in macrophages causing visceral leishmaniasis (kala-azar) in human being. The disease is an often fatal and endemic in certain region of India and some other tropical and subtropical countries around the world. ${ }^{1}$

The expression of a major phosphorylated glycoconjugates on its surface membrane i.e., lipopolysaccharide (LPPS), ${ }^{2}$ which is most commonly known as lipophosphoglycan (LPG) ${ }^{3-5}$ anchored by glycosylphosphatidylinositol (GPI) and glycosylinositolphospholipid (GIPL) and Gp63, a metaloprotease are the ligand for macrophage receptor for the attachment of the parasite, which is the initial step for infection regulated by cAMP both in parasite and macrophage. ${ }^{6}$ The parasite also expresses highly glycosylated secreted acid phosphatase, which is considered beneficial by dephosphorylating a wide spectrum of bioactive molecules ${ }^{7,8}$ in both the environment encountered in its life cycle. Regulation of parasite physiology essential for the survival in hostile environments of host or vector in two distinct morphological forms is largely depend on signal transduction systems.

Cyclic AMP inhibited the growth of Leishmania promastigotes ${ }^{9,10}$ but the nature of its action remains tobe elucidated. Intracellular cAMP possibly involves in signal transduction in Leishmania promastigotes. The mRNAs of a family of putative receptor adenylyl cyclases are expressed in insect stage of $L$. donovani possibly involved in the increase of intracellular cAMP ${ }^{11}$ the necessity of which is yet to be determined. The parasite exposes to the presence of intracellular cAMP modifying agents e.g. epinephrine, norepinephrine in host as

Correspondence: Dr Kalipada Kar, Department of Physiology, College of Medical Sciences and Teaching Hospital, Bharatpur, Nepal. E-mail: kalipadakar@hotmail.com. DOI: 10.3126/jcmsn.v\%vi\%i.19380. Article received: 2018-03-13. Article accepted: 2018-06-26. 
well as in vectors blood meal. However, the role of adrenergic receptor agonists and antagonists in the growth of $L$. donovani promastigotes is yet to be elicited.

In this report, we studied the effects of membrane permeable cAMP and some adrenergic receptor agonists and antagonist of intracellular cAMP formation on the growth of $L$. donovani promastigotes.

\section{MATERIALS AND METHODS}

Adenine, hemin, D-biotin, L-glutamine, folic acid, epinephrine, isoproterenol, propranolol, dibutyryl cycle GMP (db-cGMP) and dibutyryl cycle AMP (db-cAMP) from Sigma Co., USA; medium 199, HEPES and beef heart infusion (BHI) from Gibco Co., USA and agar from Difco Co., USA were purchased for the experiments. Radioactive $\left[{ }^{32} \mathrm{P}\right]$ orthophosphate was obtained from BARC, India. Other reagent grade chemicals were used in the study.

Parasite maintenance and effects of experimental agents on promastigote stage: Promastigotes of L. donovani (MHOM/IN/78/ UR6), ${ }^{12}$ an Indian strain subpassaged in solid blood agar medium consisting of BHI $(3.7 \%, \mathrm{w} / \mathrm{v})$, Dglucose $(1.8 \%, \mathrm{w} / \mathrm{v})$, agar $(1.5 \%, \mathrm{w} / \mathrm{v})$ and rabbit blood $(2 \%, \mathrm{v} / \mathrm{v})$ grown at $24 \pm 1^{\circ} \mathrm{C}$ was used in this study. The parasite was then cultured in S1-199 medium, ${ }^{13} \mathrm{pH} 7.4$ composed of medium 199 fortified with $25 \mu \mathrm{M}$ HEPES, $4 \mu \mathrm{M}$ 1-glutamine, 10 $\mu \mathrm{M}$ D-glucose, $0.6 \mu \mathrm{M}$ adenine and $8 \mu \mathrm{M}$ hemin with or without experimental agents at $24 \pm 1^{\circ} \mathrm{C}$ for 144 hour. The growth profiles were recorded by counting promastigotes present in aseptically withdrawn $20 \mu 1$ sample from each experimental set diluting in formol-saline ( $4 \%$ formol) in a ratio of $1: 1$ for early period of culture or $1: 10$ as depending on the parasite concentration using a hemocytometer and the results were expressed as the mean of four separate experiments as described. ${ }^{13}$

\section{Biosynthetically pulse labeling of the promastigotes with $\left[{ }^{32} \mathrm{P}\right]$-orthophosphate and its analysis:}

Promastigotes of log phase culture in S1-199 medium were washed twice with sterile saline $(0.85 \%, \mathrm{w} / \mathrm{v})$ by centrifugation at $1000 \mathrm{xg}$ for 10 min at $4{ }^{\circ} \mathrm{C}$. These washed parasites were allowed to grow in the fresh $\mathrm{S} 1-199$ medium to which $\left[{ }^{32} \mathrm{P}\right]$ $(50 \mu \mathrm{Ci} / \mathrm{ml}$ of culture) was added in presence or absence of experimental agents. Initial parasite concentration was adjusted to $2.0 \times 10^{7} / \mathrm{ml}$. After $6 \mathrm{~h}$ of incubation at $24 \pm 1{ }^{\circ} \mathrm{C}$ the parasites were harvested by centrifugation in eppendorf centrifuge and washed thrice with phosphate buffered saline, $\mathrm{pH} 7.2$, at $4{ }^{\circ} \mathrm{C}$. Immediately, the $\left[{ }^{32} \mathrm{P}\right]$-labeled parasites were suspended in denaturing sample buffer (1X, final) and boiled for $5 \mathrm{~min}$ at $100{ }^{\circ} \mathrm{C}$ and the macromolecules were subjected to separation by SDS-PAGE, Coomassie Brilliant Blue stained, dried and followed by autoradiography using Kodak X-Omat AR film with intensifying screen at $70^{\circ} \mathrm{C}$. Statistical analysis: The results of the experiments were tested for statistical significance using the Student $t$ test using Microsoft excel, Microsoft Corporation. Comparisons were made between the control (no drug) and each experimental set containing different drugs at different concentrations and further in between combination group and individual agent of that group. $\mathrm{P}$ value of $<0.05$ was considered as statistically significant.

\section{RESULTS}

The effect of db-cAMP, db-cGMP, epinephrine, isoproterenol and propranolol on the growth of L. donovani promastigotes:

The growth curve of promastigotes in control was presented in Fig. 1 as inset based on mean with SD value of four identical experiments for each time point. The results obtained with experimental agents were also treated in the same way to determine the means, SD values and $\mathrm{P}$ values. The effects of experimental agents were expressed as percent of stimulation or inhibition in compared to growth in control at $72 \mathrm{~h}$ and $96 \mathrm{~h}$ incubation were shown in figure 1. Although the trends of effect of these compounds on cell division were very similar at $72 \mathrm{~h}$ and $96 \mathrm{~h}$ culture, it was more prominent in $96 \mathrm{~h}$ as shown in figure 1. Multiplication of $L$. donovani promastigotes was inhibited by $16 \%$ $(\mathrm{P}<0.01), 28 \% \quad(\mathrm{p}<0.001), 43 \% \quad(\mathrm{P}<0.0005)$ and $69 \%(\mathrm{P}<0.0001)$ at $96 \mathrm{~h}$ culture by $10,20,50$ and $100 \mu \mathrm{M}$ db-cAMP compared to control, respectively (Fig-1A). On the contrary the activity of db-cGMP on the growth of the parasite was found significantly stimulatory by $15 \%(\mathrm{p}<0.005)$, $41 \% \quad(\mathrm{P}<0.0005), \quad 49 \% \quad(\mathrm{P}<0.0001) \quad$ and $\quad 50 \%$ $(\mathrm{P}<0.0001)$ with $10,20,50$ and $100 \mu \mathrm{M}$ of the agent, respectively, at $96 \mathrm{~h}$ culture.

The growth of the parasite was drastically inhibited by epinephrine in a dose dependent manner. The inhibition was posted by $20 \%(\mathrm{P}<0.001), 38 \%$ $(\mathrm{P}<0.0005), 59 \%(\mathrm{P}<0.0001)$, and $83 \%(\mathrm{P}<0.0001)$ with $10,20,50$ and $100 \mu \mathrm{M}$ epinephrine, 
Kar et al. Leishmania Donovani Promastigote: Effect of Adrenergic Agonists and Antagonist..

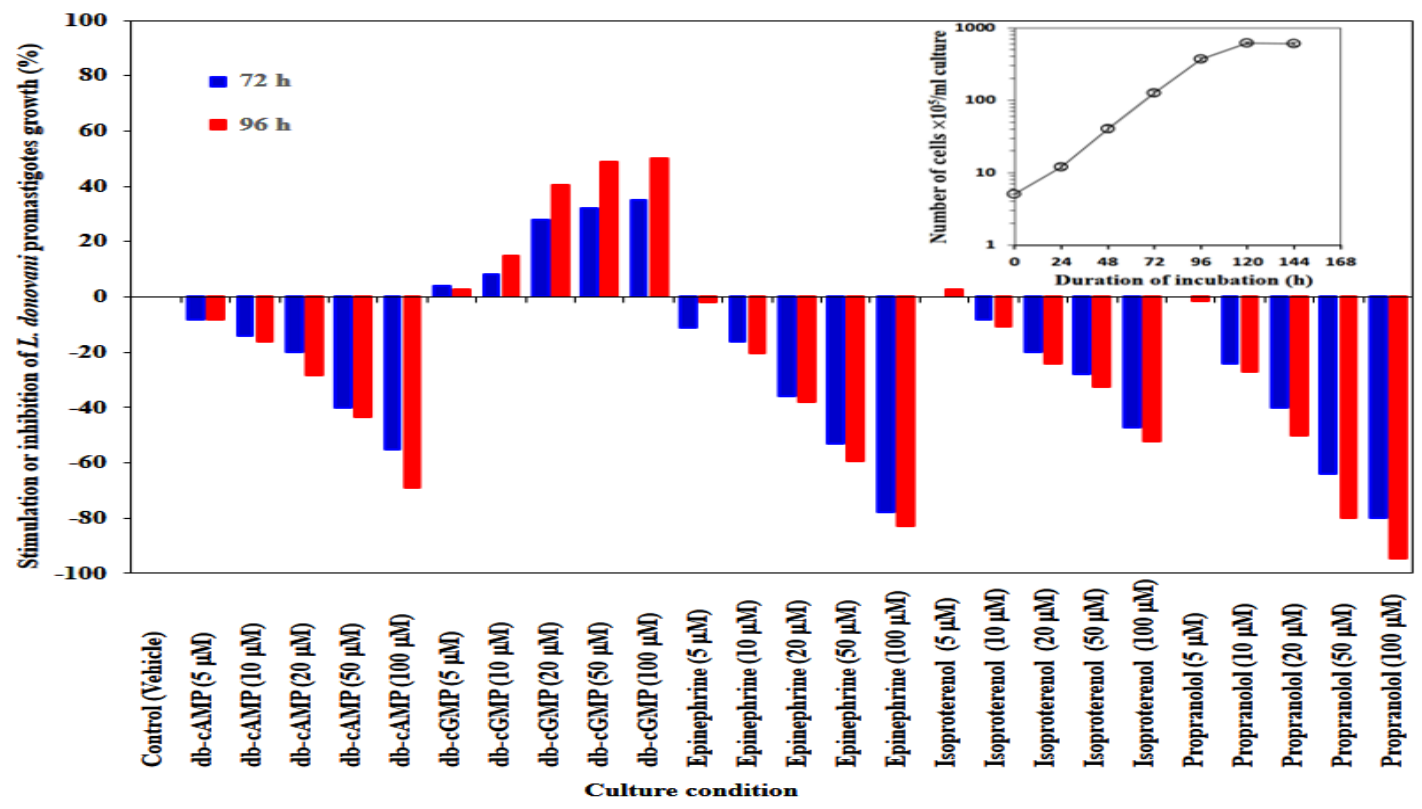

Figure 1. Histograms show the percent of stimulation or inhibition of growth of $L$. donovani promastigotes treated with experimental agents for $72 \mathrm{~h}$ (blue bar) or $96 \mathrm{~h}$ (red bar), where, the growth of the parasite in control (no drug) was considered as zero at respective time points of culture based on the mean of four separate identical experiments. The growth curve with error bar $( \pm \mathrm{SD}, \mathrm{N}=4)$ for control was presented as inset where each point stands for mean of four separate identical experiments. The experimental agents and their different concentrations were mentioned below for each pair of histogram for $72 \mathrm{~h}$ and $96 \mathrm{~h}$ culture, respectively.

respectively at $96 \mathrm{~h}$ culture and the similar pattern of inhibition was recorded at $72 \mathrm{~h}$ culture (Fig.1). We tested the effect different doses of isoproterenol, an agonist of $\beta$-adrenergic receptor on the growth of promastigote and found $11 \%$ $(\mathrm{P}<05), 24 \%(\mathrm{P}<0.005), 32 \%(\mathrm{P}<0.001)$ and $52 \%$ $(\mathrm{P}<0.0001)$ inhibition with $10,20,50$ and $100 \mu \mathrm{M}$ of the drug, respectively.

The effect of dibutyryl cAMP, epinephrine, isoproterenol and propranolol in the phosphorylated macromolecules LPG profile of L. donovani promastigotes: Regulation of LPG in L. donovani promastigotes by cAMP, epinephrine, isoproterenol and propranolol were visualized by autoradiography after SDS-PAGE analysis of $\left[{ }^{32} \mathrm{P}\right]-$ labeled parasite in presence or absence of these agents individually for $6 \mathrm{~h}$. A typical experimental result of three replicate experiments was shown in figure 2. There was no prominent change in protein profiles of promastigotes treated with or without experimental agents (Figure 2B. $\mathrm{a}^{\prime}-\mathrm{e}^{\prime}$ ). In control a heterogeneous macromolecule spanned in approx. 28-40 $\mathrm{kDa}$ region of the gel (Figure 2A. Lane a) that was leishmanial lipophosphopolysaccharide (LPPS), which is known as LPG confirmed by phenol extraction of the metabolically phosphatelabeled whole cell and analyzed by SDS-PAGE and autoradiography as described by Kar et al. $1991^{2}$ (Fig. not Shown). The reduction in the intensity of

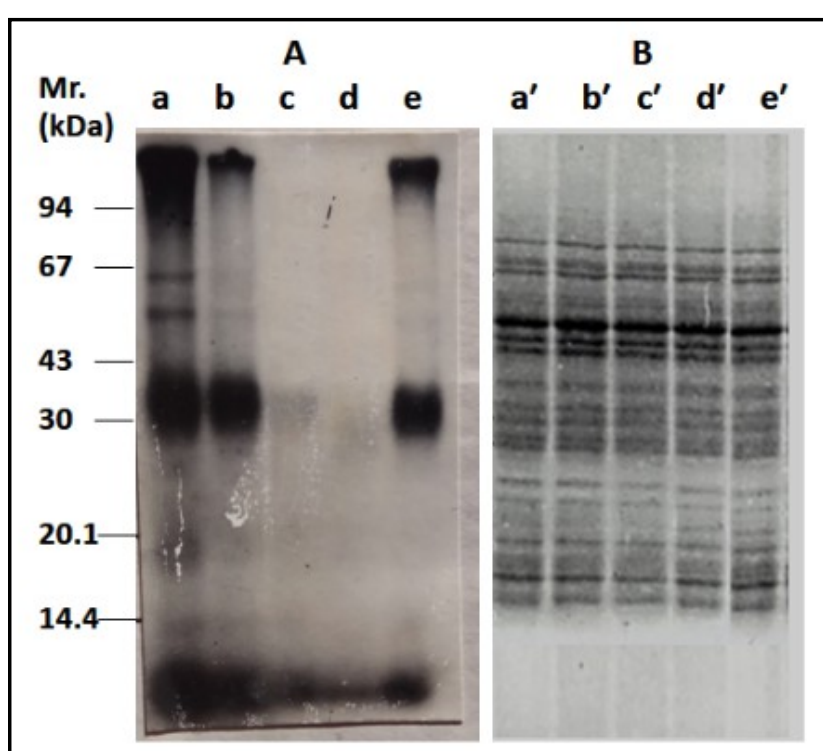

Figure 2. Autoradiograph after SDS-PAGE (12.5\% acrylamide) analysis of the effect of agonists and antagonists of $b$-blocker on the $\left[{ }^{32} \mathrm{P}\right]$-phosphorylation of $L$. donovani UR6 promastigotes was shown in figure 2A. Equal number of $\left[{ }^{32} \mathrm{P}\right]$-labelled UR6 promastigotes $\left(1 \times 10^{7}\right)$ for each sample were loaded per lane. Lane a, control (without any drug); lane b, db-cAMP (100 $\mu \mathrm{M})$; lane c, adrenaline $(100 \mu M)$; lane d, propranolol $(100 \mu \mathrm{M})$ and lane $e$, isoproterenol $(100 \mu \mathrm{M})$. The corresponding protein profiles were shown in Fig $2 B$ : lane $a^{\prime}, b^{\prime}, c^{\prime}, d^{\prime} \& e^{\prime}$ for control, db-cAMP, adrenaline, propranolol and isoproterenol, respectively. 
the band observed in the autoradiograph from higher to lower was registered with the agents in the following manner: propranolol $>$ epinephrine $>$ cAMP > isoproterenol at $100 \mu \mathrm{M}$ each (Figure 2A. Lane d, c, b \& e, respectively).

\section{DISCUSSIONS}

Intracellular levels of cAMP have been demonstrated to modulate metabolism and replication of prokaryotic ${ }^{14}$ and eukaryotic cells. ${ }^{15}$ It was reported that the growth of L. tropica and $L$. donovani were inhibited by $70 \%$ with dibutyryl cAMP at $1 \mu \mathrm{M} .{ }^{16}$ We have found the same degree of inhibition with one tenth of that concentration of the agent. This difference obtained may be due to the strain variation and/or culture condition. The variation may be attributed to the difference in the level of cAMP phosphodiesterase activity which is essential for the down regulation of cAMP. ${ }^{17}$

It is prominent that the growth stimulatory effect was observed with the gradual increase of $\mathrm{db}$ cGMP upto $50 \mu \mathrm{M}$, and beyond this concentration there was no more beneficial to the parasite may be due to the limitation of the culture medium as a whole. Availability of cGMP in a large amount from external source was found beneficial to Leishmania promastigotes may be attributed to modulate some intracellular signaling, which is different than the effect of cAMP and/or serve as added purine source as the purine salvage pathway is active in Leishmania $^{18}$ and the parasite essentially expresses enzymes including phosphatases and nucleases for scavenging purine. $^{19}$

It is evident from our results that the parasite physiology was significantly more modulated by epinephrine than isoproterenol and this may be vested on both $\alpha$ and $\beta$ adrenergic receptor specificity of epinephrine whereas isoproterenol is an only $\beta 1 \& \beta 2$ adrenergic receptors agonist.

Leishmania LPG, is a highly complex macromolecule composed of four distinct domains: a GPI anchor consists of an alkyl phosphatidylinositol having a single saturated C24- 26 aliphatic chain; ${ }^{20}$ a glycan core consists of a heptasaccharide comprising two galactopyranosides, a galactofuranoside (Galf), two mannosides and a glucosamine residue attached to inositol; a linear phosphoglycan chain (PG) consists of 15-40 phosphodisaccharide (Galß1,4Mano1-PO4) units; and finally, LPG is terminated by a oligosaccharide cap having di-, trior tetrasaccharide consisting of galactose and mannose assembled as Man $\alpha 1,2$ Man $\alpha 1$ or as Gal $\beta 1,4$ (Man $\alpha 1,2$ ) Man $\alpha 1$ depending on the Leishmania species. ${ }^{5}$ It is clear from our results that at least some of the steps in the synthesis of LPG are affected by cAMP. The reduced infectivity of promastogotes to macrophage by cAMP was documented ${ }^{6}$ and this is possibly due to the reduced LPG expression and affecting other cellular physiology of the parasite by cAMP and its modifiers.

This study has clearly shown that the synthesis of LPG, the major glycoconjugates of $L$. donovani was controlled by cAMP and significantly modulated by adrenergic receptor agonists epinephrine, which is effective on both $\alpha$ - and $\beta$ receptors than isoproterenol, a nonselective $\beta 1$ adrenoreceptor agonist which is well known for contraction of cardiac muscle by opening of calcium channel and dilatation of smooth muscle by closing of calcium channel via $\beta 2$-receptor. Propranolol is a $\beta 1$ and $\beta 2$ adrenoreceptors antagonist and has membrane stabilizing activity. Propranolol significantly affects the replication of $L$. donovani promastigote and synthesis of LPG. However, the precise nature of activity of propranolol on Leishmania promastigotes is yet to be determined.

\section{CONCLUSION}

This report describes the role of cAMP, epinephrine, isoproterenol and propranolol in Leishmania donovani promastigotes. The growth of $L$. donovani promastigotes at $96 \mathrm{~h}$ culture was inhibited by $69 \%, 83,52 \%$ and $95 \%$ with dbcAMP, epinephrine, isoproterenol and propranolol at $100 \mu \mathrm{M}$ each, respectively in compared to control. The similar patterns of growth inhibition of the parasite for all the agents were also observed at $72 \mathrm{~h}$ culture. Multiplication of parasite was stimulated by db-cGMP. The maximum growth stimulatory effect was achieved with $50 \mu \mathrm{M}$ dbcGMP. The expression of LPG of the parasite was drastically affected with propranolol and epinephrine in compare to cAMP or isoproterenol at $100 \mu \mathrm{M}$ of each agent for $6 \mathrm{~h}$ exposer to the promastigotes in defined culture medium, whereas the protein profiles apparently remain identical with the control.

\section{ACKNOWLEDGEMENT}

The entire experimental work was done in the Indian Institute of Chemical Biology, Kolkata, India, during 1989 to 1997. Data interpretation and manuscript writing were done during our stay in 
Kar et al. Leishmania Donovani Promastigote: Effect of Adrenergic Agonists and Antagonist..

College of Medical Science-TH, Bharatpur, Chitwan, Nepal. Authors are grateful to Late Prof. A.N. Bhaduri and Late Dr. J. Das, Ex-Directors, and Dr. D.K. Ghosh, Ex-Asst. Director, Indian Institute of Chemical Biology, Kolkata 700032,

\section{REFERENCES}

1. Kar K. Serodiagnosis of Leishmaniasis. Crit Rev Microbiol.1995; 21: 123

2. Kar K, Mukherji K, Kar S, Sarkar D, Bhattacharya A, Ghosh DK. Biochemical and immunological characterization of exometabolites from an Indian strain of Leishmania donovani promastigotes grown in a chemically defined medium. MolCell Biochem. 1991;108:159

3. Favila MA, Geraci NS, Jayakumar A, Hickerson S, Mostrom J, Turco SJ, et al. Differential Impact of LPG-and PG-Deficient Leishmania major Mutants on the Immune Response of Human Dendritic Cells. PLoSNegl Trop Dis. 2015; 9 (12): e 0004238. doi:10.1371/ journal.pntd.0004238

4. Forestier CL, Gao Q, Boons GJ. (2015). Leishmania lipophosphoglycan: how to establish the structure-activity relationships for this highly complex and multifunctional glycoconjugate? Frontier in Cell Infect Microbiol. 2015; 4193. doi: 10.3389/fcimb.2014.00193. eCollection 2014.

5. Turco SJ, Descoteaux A. The lipophosphoglycan of Leishmania parasites. Annu Rev Microbiol. 1992; 46:65

6. Mitra S, Ghosh L, Chakrabarty P, Biswas M, Bhattacharyya FK, Ghosh DK. Effect of bioamines on uptake of promastigotes of Leishmania donovani by hamster peritoneal macrophages J. Med. Microbiol. 1992;36: 283

7. Bates PA, Herms I, Dwyer M. Leishmania donovani: immunochemical localization and secretory mechanism of soluble acid phosphatase. Exp Parasitol. 1989; 68: 335

8. Fernandes ACS, Soares DC, Saraiva EM, Meyer -Fernandes JR, Souto-Padron T. Different secreted phosphatase activities in Leishmania amazonensis. FEMS Microbiol Lett. 2013; 340: 117 128 DOI:10.1111/1574-6968.12080.

9. Walter, R.D.(1981):Regulation of cyclic AMP metabolism in Leishmania promastigotes and amastigotes. In: Biochemistry of Parasitesa: M. Slutzky, ed.), Pergamon Press, Oxford and New York, pp 151-167.
India, for providing facilities for part of this work. We are thankful to Dr. V. Natraj Prasad and Dr. M Mathur for their support. Our special thanks go to Dr. Manohar Pradhan for his constant encouragement for preparing this manuscript.

10. Bhattacharya A, Biswas A. Das PK. Role of intracellular cAMP in differentiation-coupled induction of resistance against oxidative damage in Leishmania donovani. Free Rad Biol Med.2008; 44: 779

11. Sanchez MA, Zeoli D, Klamo EM, Kavanaugh MP, Landfear SM. A family of putative receptoradenylate cyclases from Leishmania donovani. $\mathrm{J}$ Biol Chem. 1995; 270: 17551

12. Kar K. Folic acid the essential supplement to brain heart infusion broth for cultivation and cloning of Leishmania donovani promastigotes. Parasitology 1997;115: 231

13. Kar K, Mukherji K, Bhattacharya A. Ghosh DK. Cultivation of Leishmania donovani promastigotes in supplemented defined tissue culture media. Med. Sci. Res. (Biochem.) 1987;15:619

14. Mullick U. Herrlich P. Regulation of synthesis of a major outer membrane protein: cyclic AMP represses Escherichia coli protein III synthesis. Proc. Natl. Acad. Sci. U.S.A., 1979;76:5520.

15. Pastan IH. Jonon GS .Anderson WB. Role of cyclic nucleotides in growth control. Ann. Rev.Biochem.1975;44:419

16. al-Chalabi KA, Ziz LA, al-Khayat B. Presence and properties of cAMP phosphodiesterase from promastigote forms of Leishmania tropica and Leishmania donovani. Comp Biochem Physiol B. 1989;93(4):789.

17. Johner A, Kunz S, Linder M, Shakur Y and Seebeck T. Cyclic nucleotide specific phosphodiesterase of Leishmania major. BMC Microbiol. 2006; $6: 25$

18. Marr JJ, et al. Purine metabolism in Leishmania donovani and Leishmania braziliensis. Biochim Biophys Acta. 1978; 544:360-371. [PubMed: 719006]

19. Martin JL, Yates PA, Soysa R, Alfaro JF, Yang $\mathrm{F}$, et al. Metabolic Reprogramming during Purine Stress in the Protozoan Pathogen Leishmania donovani. PLoS Pathog. 2014; 10 (2): e1003938. doi:10.1371/journal.ppat.1003938

20. Ferguson MA. The structure, biosynthesis and functions of glycosylphosphatidylinositol anchors, and the contributions of trypanosome research. J Cell Sci. 1999; 112:2799

Citation: Kar K, Kar S. Leishmania Donovani Promastigote: Effect of Adrenergic Agonists and Antagonist on Growth and Lipophosphoglycan Synthesis. JCMS Nepal. 2018;14(2):111-5. 